\title{
Difficulties in identifying developmental defects of the enamel: a BITA study
}

\author{
B. Jälevik ${ }^{1}$ (D) A. Szigyarto-Matei ${ }^{2} \cdot$ A. Robertson ${ }^{1}$
}

Received: 31 May 2018 / Accepted: 11 March 2019 / Published online: 18 March 2019

(c) The Author(s) 2019

\begin{abstract}
Aim To evaluate the ability to recognise different types of developmental defects of enamel (DDE) by a group of general dental staff, trained prior to the comprehensive prevalence study (the BITA study), and to compare their skills to that of an untrained group. To evaluate the validity and reliability of the DDE registrations and MIH diagnoses, 3 years after the BITA study ended. To evaluate the reliability of an MIH diagnose, 3 years after the study ended.

Materials and methods The dental staff from five clinics $(n=43)$ was given instructions in identifying different types of DDE, according to the mDDE index (FDI in Int Dent J 42:411-426 1992). A test protocol with 24 digital photos of teeth with different DDE was filled out. The dental staff from five other clinics $(n=60)$, without preceding instructions, also filled out the protocol. Ninety-one patients with reported DDE were clinically re-examined 3 years later by two dentists with certified experience of DDE.

Results When untrained dental staff filled out the test protocol, only $42 \%$ of the affected surfaces were correctly assessed, whereas $85 \%$ of the dental staff instructed in recognizing DDE made a correct assessment $(p<0.000)$. The specialists confirmed $73 \%$ of the teeth diagnosed with DDE in the BITA study as affected. The agreement in judging the opacities as diffuse or demarcated was $76 \%$. One-fourth of those being judged to be affected by MIH in the BITA-study also showed to be affected by other enamel disturbances.

Conclusions The ability of the untrained dental staff to recognize DDE was insufficient. Instructions, using digital photos, raised the ability considerably. To ensure a correct MIH diagnosis, the patients should be followed until all the permanent teeth have erupted.
\end{abstract}

Keywords Enamel $\cdot$ Hypomineralisation $\cdot$ Hypoplasia $\cdot$ Identification

\section{Introduction}

Developmental defects of the enamel (DDE) are common in many child populations (King 1989; Dummer et al. 1990; Elley and Charlton 1992). They may occur as pits or grooves, denominated hypoplasia, and are defined as a quantitative defect of enamel or as a white or discoloured area, denominated opacities, and are defined as a qualitative defect, i.e., an enamel hypomineralisation. There are

B. Jälevik

birgitta.jalevik@gu.se

1 Department of Pediatric Dentistry, Institute of Odontology at the Sahlgrenska Academy, University of Gothenburg, Gothenburg, Sweden

2 Specialist Clinics of Pediatric Dentistry, Public Dental Service, VGR, Uddevalla, Sweden two kinds of opacities; demarcated opacities have a distinct and clear boundary to the adjacent normal enamel, and diffuse opacities have a linear, patchy, snow-capped, cloudlike, or confluent distribution, but with no clear boundary to the adjacent normal enamel. The defects may be localised, affecting single or multiple teeth or generalised, either affecting groups of teeth developing at the period of disturbance, or most teeth independent of the time of development (The mDDE index FDI 1992; Espelid et al. 2017).

DDE affects individuals to varying degrees. All teeth or just single teeth can be affected. The enamel can be soft and disintegrating, or with just a few discreet opacities. The patients can be exposed to great suffering with pain, difficulties in eating or tooth brushing, but also with great aesthetical problems (Jälevik and Klingberg 2002), or have hardly noticed the defects at all. 
The manifestation of DDE can be pathognomonic for a specific affection, e.g., fluorosis (Fejerskov et al. 1996; DenBesten 1999) and MIH (Jälevik and Norén 2000; Fagrell et al. 2010; Elhennaway et al. 2017), while in other cases, the clinical appearance does not reveal the causation (Small and Murray 1978). The clinical appearance of fluorosis (Fejerskov et al. 1996), as well as the appearance of MIH (Weerheim et al. 2003), is well described and also defined in a histological aspect (Fejerskov et al. 1996; Elhennaway et al. 2017). Nevertheless, the clinical view can be mixed up with other types of DDE in the clinic. Sabokseir et al. (2016) suggest that one-fourth of a group of children diagnosed with fluorosis had non-fluoride-related DDEs resembling fluorosis.

The reported prevalence of MIH varies considerably, 2.4-40.2\% (Jälevik 2010), indicating differences in recognising MIH. The mild defects may be particularly hard to discern from other defects.

A correct diagnosis is essential when making a treatment plan and also when searching for the aetiology for MIH. The type of defect (hypoplasia, demarcated or diffuse opacities) and their spreading in the mouth must be taken into consideration.

The aims with this study were:

\section{Part 1}

- To evaluate the ability of the dental staff in general dentistry to recognise different types of DDE.

- To evaluate the ability to recognise different types of DDE in a trained group of general dental staff, compared to an untrained group.

\section{Part 2}

- To evaluate the validity and reliability of the DDE registrations of the trained, general dental staff in a clinical follow-up study after 3 years.

- To investigate the presence of MIH, based on DDE registrations in the BITA study, and to compare this with the findings in the clinical follow-up.

\section{Materials and methods}

\section{Part 1}

\section{Trained group}

As preparation for a 5-year longitudinal study in Sweden [the BITA study concerning different aspects in the oral health of children (Oldin et al. 2015)], the staff in five Public Dental Service clinics in the Region Västra Götaland were given a 2-h lecture on how to recognise developmental defects of the enamel (DDE). They were trained in discerning between demarcated opacities (DEO), diffuse opacities (DIO), and hypoplasia (Hypo), according to the mDDEindex (FDI 1992). They were also informed on more common diagnoses such as fluorosis, MIH, and amelogenesis imperfecta. Digital photos were used in the instructions.

Two weeks later, the participants filled out a test protocol with 24 digital photos of teeth with different DDE, shown on a computer screen. Thirty-four affected surfaces were available to judge. All personnel $(n=43)$, consisting of dentists $(n=20)$, dental hygienists $(n=5)$, and dental assistants $(n=18)$, participated. The tests were repeated 2 years later with the same photos in a different order to control the reliability and validity of the registrations.

\section{Control group}

The dental staff in five other clinics $(n=60)$, consisting of dentists $(n=29)$, dental hygienists $(n=13)$, and dental assistants $(n=18)$, filled out the test protocol. They were only told to discern between demarcated opacities, diffuse opacities, and hypoplasia, with no other instructions.

\section{Part 2}

After the BITA study ended, 264 had been diagnosed with DDE. The prevalence and distribution of the different types of DDE were reported (Jälevik et al. 2018). 201 children accepted further participation in a follow-up study with a complementary questionnaire after the study ended, jointly filled out by the children/adolescents and their caretakers (Jälevik et al. 2018). Two percent reported a somewhat raised fluoride level in their drinking water. No correlation was found between early health problems, reported in the BITA study, and DDE (Jälevik et al. 2018). Three years after the BITA study ended, 100 participants were randomly selected to be re-examined in the clinic by two experienced paediatric dentists (BJ, ASM). The study cohorts were 14, 18 and 22 years old at the clinical follow-up examination.

The DDE in all erupted teeth were registered and clinical digital photos [a "five-view set" including frontal, two lateral views plus superior and inferior views (Wong et al. 2005)] were taken (camera: Nikon D90/80, lens: Nikon AF-S-Micro-Nikkor 85 mm Nikon DX, macro-flash: Sigma EM-140 DG NA).

After KAPPA calculation (0.82), the paediatric dentists came to an agreement on the types of DDE in each tooth by a joint assessment, based on their clinical registrations and photos. In each individual, the distribution of DDE (local, chronological, or non-chronological), as well as the fulfilment of the MIH criteria, was assessed. According to the MIH criteria, the permanent first molars and incisors, as 
index teeth, were controlled for the absence or presence of demarcated opacities. Information on post-eruptive enamel breakdown, atypical restoration, and extraction was collected from the dental records and $\mathrm{X}$-rays. To be judged as $\mathrm{MIH}$, at least one first permanent molar had to be affected by demarcated opacities. The clinical findings concerning whether a tooth was affected by DDE, the type of DDE, and the distribution of the defects within the jaws were then compared to the original BITA study findings.

\section{Analysis}

Data were entered into a spreadsheet (Microsoft Excel, Microsoft Corp, WA, USA) and then analyzed using SPSS Version 22 (SPSS Inc, Chicago IL, USA). Comparisons between different dental groups were made with the chisquare test. Results at an alpha level less than 0.05 were considered statistically significant.

\section{Ethical considerations}

BITA-study: application for an ethical review (Dnr: 286-07) was submitted in June 2007. The Regional Ethical Review Board of the University of Gothenburg, Sweden, found the project was not subject to the Swedish Act on Ethical Review. The Board provided feedback on the information to the patients, which was considered during further planning of the study. The caretakers and their children received a letter with information regarding the project before entering the BITA-study. Respondents were asked to participate in the study when they attended a routine dental appointment. In connection to the first clinical examination, the caretakers signed an informed consent to participate in the research project.

Questionnaire: the study was approved by the Regional Ethical Review Board of the University of Gothenburg,
Sweden, Dnr: 806-13 (2014-02-25). Children/adolescents and their caretakers were given a written information regarding the study and asked to give their consent to participate.

\section{Results}

\section{Part 1}

When untrained dental staff filled out the test protocol, only $42 \%$ of the affected surfaces were correctly assessed, while $85 \%$ of the dental staff instructed in recognizing developmental defects of the enamel (DDE) made a correct assessment $(p<0.000)$ (Table 1). There was no significant difference between the first test and the repeated test of DDE in this group.

Among the trained dental staff, the ability to diagnose DDE did not differ between the dentists, the dental hygienists, and the dental assistants, whereas in the untrained group, the hygienists were less familiar with DDE than the dentist $(p=0.02)$.

The untrained staff had a marked tendency to denominate any type of DDE as hypoplasia. The trained staff showed a tendency to judge extensive demarcated opacities as diffuse opacities (Fig. 1).

\section{Part 2}

Of the 100 patients randomly selected from the BITA follow-up study, 91 were eligible for clinical examinations. The drop-outs, all in the oldest cohort, had left their home district and were not available for clinical examinations.

\section{Validity and reliability of the DDE registrations}

The specialists confirmed that $73 \%$ of the teeth diagnosed with DDE in the BITA study were affected. The agreement in judging the opacities as diffuse or demarcated was $76 \%$.
Table 1 The results from testing the ability to discern demarcated opacities, diffuse opacities, and hypoplasia

\begin{tabular}{lllllll}
\hline & $N$ & \multicolumn{3}{l}{ Correct judgement of 34 surfaces } & \\
\cline { 3 - 6 } & & Mean & $\%$ & Std & Median & Range \\
\hline Trained group test 1 & 43 & 28.89 & 85 & 4.76 & 30.5 & $14-34$ \\
Trained group test 2 & 41 & 27.93 & 82 & 4.96 & 29.5 & $13-34$ \\
Control group & 60 & 14.23 & 42 & 6.16 & 13.5 & $3-32$ \\
Test1-Ctr $p<0.000$ & & & & & & \\
Test1-Test 2 NS & & & & & & \\
\hline
\end{tabular}

In the test with 24 photos of teeth with developmental defects of the enamel, 34 surfaces were available for evaluation. The trained group had their first test 2 weeks after a lecture in developmental defects of the enamel. The second test took place years later. The control group had no lecture prior to filling out the test protocol

Ctr control, NS non-significant 
Fig. 1 Five of the photos in the test form illustrating different types of developmental defects of the enamel (DDE), and how the test group and the control group judged them, respectively. The control group had a predilection to diagnose the majority of defects as hypoplasia $(\mathbf{a}-\mathbf{e})$. The test group succeeded in discerning post-eruption from hypoplasia (e). Although the test group showed a better ability to discern different DDE, there was a tendency that extensive demarcated opacities opacities (DIO) (a, d). However, DIO was often judged as DEO in a first permanent molar with fluorosis in both groups. In a front tooth with diffuse opacities and a discoloured area, difficulties providing a correct assessment (c) (DEO) were judged as diffuse both groups had considerable

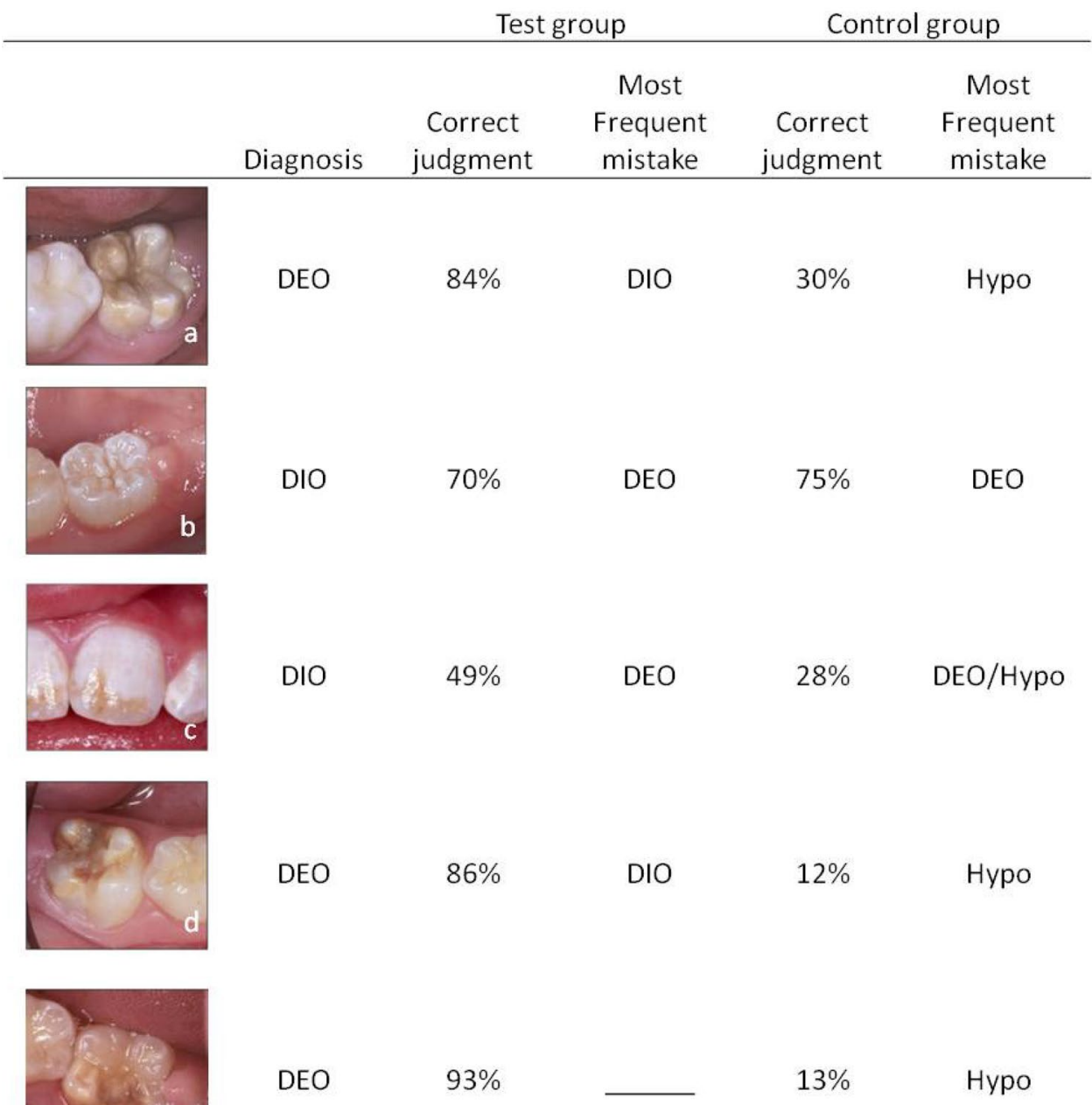

The most common discrepancy was that some teeth with demarcated opacities in the BITA study were evaluated as diffuse by the specialists, but the opposite also occurred.

Concerning the distribution of DDE within the jaws, the judgments of fully $88 \%$ of the patients agreed with the BITA study. In spite of this, one-third of them, assessed as chronological, revealed to be non-chronological in the clinical follow-up study due to the eruption of canines, premolars and/or second permanent molars.

\section{Fulfilment of MIH criteria}

In the BITA study, 97 children/adolescents $(12.2 \%$ of the total sample) were judged to fulfil the MIH criteria. Fortyfour of those who were randomly selected to participate in the clinical follow-up study fulfilled the MIH criteria. When clinically examined, it was revealed that only 33 fulfilled the MIH criteria (Table 2). Consequently, one-fourth of those judged to be affected with MIH in the BITA study showed to be affected with other enamel disturbances. Moreover,
$\mathrm{MIH}^{*}$

Table 2 Based on the registered developmental defects of the enamel (DDE) and contemporaneous information in records, an MIH diagnosis (Weerheijm et al. 2003) was made in the group of children, who 3 years later were examined and photographed in the clinic

\begin{tabular}{lrlr}
\hline Information from BITA & $N$ & $\begin{array}{l}\text { Clinical examination and } \\
\text { photos 3 years later }\end{array}$ & $N$ \\
& \multirow{2}{*}{ MIH } & MIH* & 12 \\
& & MIH* & 15 \\
& & Other enamel disorder(s) & 8 \\
& & No disorder & 1 \\
& 8 & MIH* & 2 \\
MIH* & & MIH* & 4 \\
& & Other enamel disorder(s) & 2 \\
& & No disorder & 0 \\
Other enamel disorder(s) & 47 & MIH* & 2 \\
& & MIH* & 1 \\
& & Other enamel disorder(s) & 41 \\
& & No disorder & 3 \\
\hline
\end{tabular}

$M I H^{*}$ MIH criteria fulfilled, but other teeth than first permanent molars and incisors are also affected by any type of DDE 
one-fifth of those with plausible MIH in the BITA study and half of those in the follow-up study also had additional teeth affected (canines, premolars and/or second permanent molars), than the first permanent molars and incisors. These teeth may have been affected with diffuse or demarcated opacities and in a few cases, with hypoplasia.

\section{Discussion}

The present study illustrates problems in recognizing developmental defects of the enamel (DDE).

The most striking finding in the first part of the present study is the poor ability of the untrained dental staff to recognise DDE. They seemed to lack up-to-date knowledge. Earlier dental education taught to denominate all enamel defects as hypoplasia. As a consequence, many in the dental staff had limited knowledge to discern different types (Fig. 1). Hubbard et al. (2017) in the D3 Group emphasized this weakness in observations of mineralisation disturbances of the enamel.

A brief education seemed to improve this ability considerably. The trained group showed good capacity to identify DDE and to discern the different types in the digital photographs. The re-testing 2 years later showed that the ability in recognising DDE remained. Digital photos were used in teaching and in testing for knowledge of DDE, as it has been shown to be a reliable method (Wong et al. 2005; Elfrink et al. 2009).

In the second part of the present study, the clinical examinations aimed to explore the reliability and validity of the BITA registrations over time.

The validity of the registrations of teeth with DDE of any type within the BITA study was fairly good. However, one-fourth of the diagnosed teeth were given a clean bill of health from DDE in the clinical follow-up. One possible reason is that DDE, especially in mild defects, tends to "fade" with time, in particular the superficial fluorotic defects due to attrition, abrasion and erosion (Wong et al. 2016). Mild opacities may become less distinct with time due to the maturing and staining of the surrounding enamel.

Even though there was an agreement about the type of DDE in most of the cases, one-fourth of the teeth were reassessed in the clinical follow-up. The most common disagreement was that the first permanent molars (FPM), judged to be affected by demarcated opacities in the BITA study, were reassessed to have diffuse opacities in the follow-up study, where more teeth with diffuse opacities had erupted. Teeth with a generalized "snow-capped" or "patchy-cloudlike" appearance had been judged as demarcated opacities in the BITA study, when only a few permanent teeth were erupted. Especially patchy-cloud-like defects can be taken for demarcated effects and mixed up with mild MIH. The
MIH opacities have a specific, histomorphological appearance with distinct borders to the adjacent, normally mineralised enamel (Jälevik and Norén 2000). These cloud-like, mild opacities have more diffuse borders, but there are cases where these defects are hard to distinguish in the clinic. A histomorphological examination would be desirable.

As a consequence of the reassessed FPM, the prevalence of MIH declined in the clinical follow-up. A recent metaanalysis, based on 70 studies on MIH prevalence, showed that children $<10$ years of age had a higher prevalence than children $>10$ years of age. An increasing breakdown with increasing age and subsequent problems in recognising demarcated opacities is an accepted explanation (Zhao et al. 2018). However, in the present study, the patients' dental history is followed longitudinally, and it can be maintained that the decline in MIH prevalence was caused by a reassessment of the type of DDE, from demarcated in the BITA study to diffuse opacities in the clinical study, 3 years later.

More than half of those with MIH also had other teeth affected. In some cases, there were canines with similar defects as in MIH. In many MIH cases, the later erupted second permanent molars and premolars also had opacities, but they were diffuse (Figs. 2, 3 and 4). These generalised, diffuse defects could be like fluorosis, but they also could show up as "snow-capped" and/or a "patchy-cloud-like" appearance (Figs. 2, 3).

The findings that MIH and other types of DDE were present simultaneously and the uncertainty in judging certain types of DDE bring about questions:

- Are teeth other than the permanent first molars, primary second molar, and permanent incisors affected by MIH? Can MIH affections show different clinical expressions?

- Are individuals affected by MIH more susceptive to other types of DDE, perhaps a genetic component?

There are still no answers to these questions. There is a need for histomorphological and biochemical examinations of teeth other than the first permanent molars extracted due to severe disturbances in the mineralisation. There is also a need for more research concerning the enamel maturation process and the rolls of the different genes in the process.

Until now, MIH in permanent first molars is a welldescribed condition in clinical as well as in chemical, mechanical, and histomorphological aspects (Jälevik and Norén 2000; Fagrell et al. 2010; Elhennaway et al. 2017). When permanent incisors have been impaired simultaneously as the molars, having clinically similar defects, it has been assumed that they were affected by the same enamel disorder. The denomination MIH was proposed (Weerheijm et al. 2001) and defined as "Hypomineralisation of systemic origin of one to four first permanent molars frequently associated with affected incisors" (Weerheim et al. 2003). 

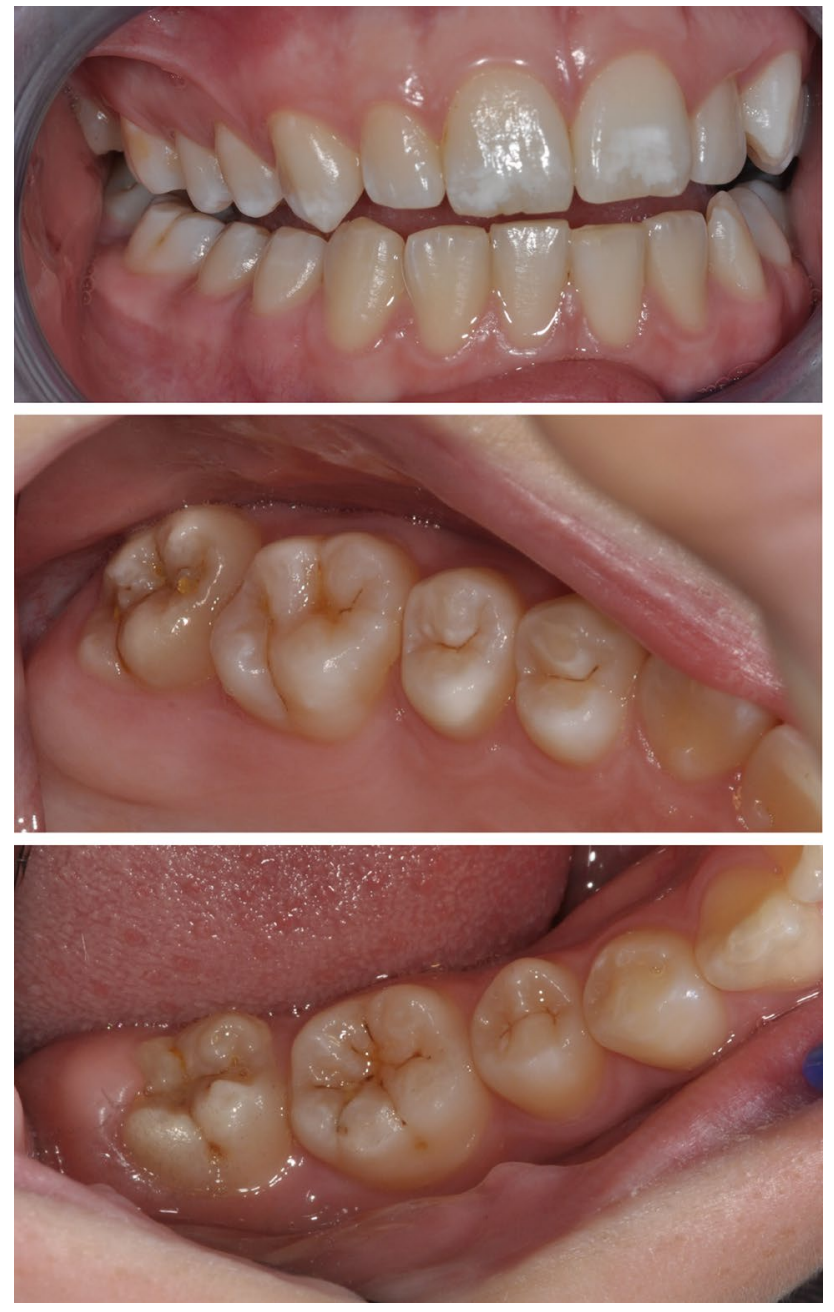

Fig. 2 A 14-year-old girl with non-chronological, cloud-like diffuse opacities. At the BITA study end 3 years earlier, she was judged to have demarcated opacities in the first permanent molars and upper central incisors

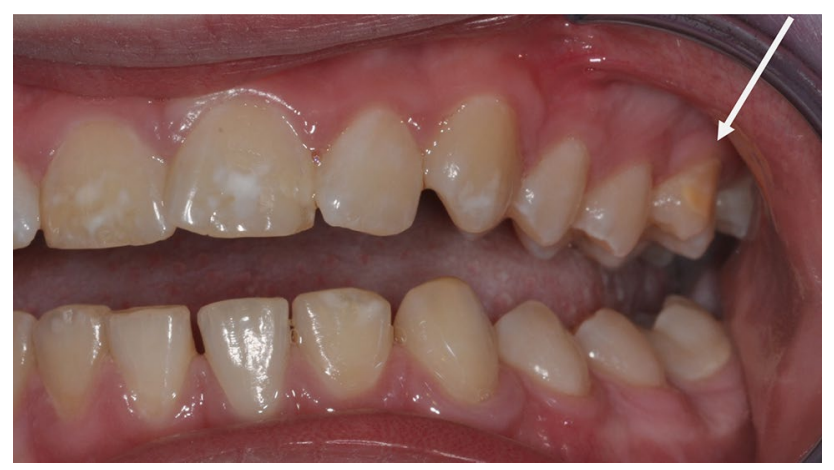

Fig. 3 A 18-year-old boy with non-chronological, cloud-like diffuse opacities and demarcated opacities in the upper first permanent molars (see arrow). The lower first permanent molars were extracted due to severe MIH and disintegration. At the BITA study end 3 years earlier, most teeth were judged to be affected by demarcated opacities

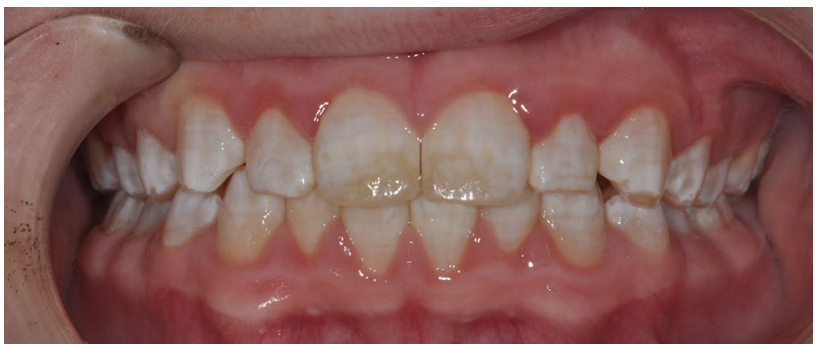

Fig. 4 14-Year-old boy. Drinking water from own well with known increased level of fluoride. At the BITA study end 3 years earlier, his first permanent molars and incisors were judged to be affected by demarcated opacities

The denomination MIH has been criticised, as it has been proven that other teeth could also be affected. Elfrink et al. (2012) showed that $4.4 \%$ of a child population had second primary molars impaired by hypomineralisation similar to MIH. In a study from Norway, it was shown that the tip of permanent canines was involved in $22.8 \%$ of the individuals in an MIH group, compared to $1.6 \%$ of those without MIH $(p<0.001)$ (Schmalfuss et al. 2016). The exclusive affection of permanent first molars and incisors in MIH suggests a specific window of time for the disturbance to occur. The period for maturation of the second primary molars and the tip of the permanent canines could be included in this window (Schour and Massler 1941). Thus, it is plausible that these defects have the same origin.

Recently, opinions are arguing that any teeth could be affect by MIH (Mittal and Sharma 2015; Viera and Cup 2016; Hubbard et al. 2017). It could be risky to judge every noted demarcated opacity as an "MIH defect", without taking the specific clinical features of $\mathrm{MIH}$, as well as their histomorphological appearance and microstructure, into account. Other possible diagnoses for demarcated opacities could be, e.g., infections in the primary predecessors, trauma or some type of genetic deviance, e.g., amelogenesis imperfecta.

Misjudgements of demarcated opacities such as MIH defects also lead to problems in searching for the aetiology. Great efforts have been unsuccessful in finding the aetiology of MIH. Focus has been on events affecting the child during the period of enamel maturation of the permanent first molars. An association between childhood illness and MIH has been found, but it is also maintained that the aetiology most likely is multifactorial (Alaluusua 2010; Silva et al. 2016).

Recently, attention has been given to the possible role of a genetic influence on MIH defects. Teixeira et al. (2018) have shown concordance of MIH between monozygotic twins. Viera and Cup (2016) maintained that MIH is a genetic condition, based on a case report with multiple and severe enamel defects. 
Some of the enamel defects described in the present study with patchy, cloud-like opacities spread non-chronologically could have been recognised as diffuse opacities, as well as mild demarcated opacities. These conditions could be recognised as a type of amelogenesis imperfecta, but in combination with demarcated opacities in the first permanent molars, also as a manifestation of MIH. In these cases, a genetic aetiology of MIH should be looked at. However, it is also essential to examine the microstructure of these defects and make a comparison with the specific histomorphological appearance of MIH.

The present study has shown the risk of misinterpreting some demarcated opacities as MIH, especially when only first permanent molars and incisors were erupted. These difficulties could contribute to the varying MIH prevalence figures (Jälevik 2010; Zhao et al. 2018; Schwendicke 2018).

In summary, the present study has illuminated difficulties in recognising developmental defects in the clinic. This unreliability in diagnosing brings problems when searching for aetiologic factors.

\section{Conclusions}

The ability of the dental staff in general dentistry to recognise developmental defects of the enamel (DDE) was shown to be insufficient.

A brief survey using digital photos of different DDE raised the ability considerably.

One-fourth of those who were evaluated to fulfil the $\mathrm{MIH}$ criteria at the end of the BITA study were shown to be affected by other types of DDE, 3 years later.

To ensure a correct MIH diagnosis, the children with affected first permanent molars should to be followed until the canines, premolars and second permanent molars have erupted.

\section{Compliance with ethical standards}

Conflict of interest Author B. Jälevik declares that she has no conflicts of interest. Author A. Szigyarto-Matei declares that she has no conflicts of interest. Author A. Robertson declares that she has no conflicts of interest.

Ethical approval All procedures performed in studies involving human participants were in accordance with the ethical standards of the institutional research committee and with the 1964 Helsinki declaration and its later amendments, or comparable ethical standards.

Informed consent Informed consent was obtained from all individual participants (and their caretakers) included in the study.

Open Access This article is distributed under the terms of the Creative Commons Attribution 4.0 International License (http://creativeco mmons.org/licenses/by/4.0/), which permits unrestricted use, distribution, and reproduction in any medium, provided you give appropriate credit to the original author(s) and the source, provide a link to the Creative Commons license, and indicate if changes were made.

\section{References}

Alaluusua S. Aetiology of molar-incisor hypomineralisation: a systematic review. Eur Arch Paediatr Dent. 2010;11:53-8.

DenBesten PK. Mechanism and timing of fluoride effects on developing enamel. J Pub Health Dent. 1999;59:247-50.

Dummer PM, Kingdon A, Kingdon R. Prevalence and distribution by tooth type and surface of developmental defects of dental enamel in a group of 15- to 16-year-old children in South Wales. Community Dent Health. 1990;7(4):369-77.

Elfrink ME, Veerkamp JS, Aartman IH, Moll HA, Ten Cate JM. Validity of scoring caries and primary molar hypomineralization (DMH) on intraoral photographs. Eur Arch Paediatr Dent. 2009;10(Suppl 1):5-10.

Elfrink ME, ten Cate JM, Jaddoe VW, et al. Deciduous molar hypomineralization and molar incisor hypomineralization. J Dent Res. 2012;91:551-5.

Elhennawy K, Manton DJ, Crombie F, et al.. Structural, mechanical and chemical evaluation of molar-incisor hypomineralization-affected enamel: A systematic review. Arch Oral Biol. 2017;83:272-81.

Elley KM, Charlton J. Prevalence of dental enamel defects in 6, 7 and 8-year-old children resident in West Bromwich, Sandwell, UK. Community Dent Health. 1992;10:11-21.

Espelid I, Haubek D, Jälevik B. Developmental defects of the dental hard tissues and their treatment. In: Koch G, Poulsen S, Espelid I, Haubek D, editors. Paediatric demtistry - a clinical approach. Hoboken: Wiley; 2017, pp. 261-90.

Fagrell TG, Dietz W, Jälevik B, Norén JG. Chemical, mechanical and morphological properties of hypomineralized enamel of permanent first molars. Acta Odontol Scand. 2010;68:215-22.

FDI Commision on Oral Health, Research and Epidemiology. A review of the development defects of enamel index (DDE Index). Int Dent J. 1992;42:411-26.

Fejerskov O, Richards A, DenBesten P. The effect of fluoride on tooth mineralization. In: Fejerskov O, Ekstrand J, Burt BA, editors. Fluoride in dentistry. Copenhagen: Munksgaard; 1996. pp. 112-52.

Hubbard MJ, Mangum JE, Perez VA, Nervo GJ, Hall RK. Molar hypomineralisation: a call to arms for enamel researchers. Front Physiol. 2017;8:546.

Jälevik B. Prevalence and diagnosis of molar-incisor-hypomineralisation (MIH): a systematic review. Eur Arch Paediatr Dent. 2010;11:59-64.

Jälevik B, Klingberg G. Dental treatment, dental fear and behaviour management problems in children with severe enamel hypomineralization of their permanent first molars. Int J Paediatr Dent. 2002;12:24-32.

Jälevik B, Norén JG. Enamel hypomineralization of permanent first molars: a morphological study and survey of possible aetiological factors. Int J Paediatr Dent. 2000;10:278-89.

Jälevik B, Szigyarto-Matei A, Robertson A. The prevalence of developmental defects of the enamel, a prospective cohort study of adolescents in Western Sweden. A BITA Study. Eur Arch Paediatr Dent. 2018. https://doi.org/10.1007/s40368-018-0347-7.

King NM. Developmental defects of enamel in Chinese girls and boys in Hong Kong. Adv Dent Res. 1989;3:120-5.

Mittal N, Sharma BB. Hypomineralised second primary molars: prevalence, defect characteristics and possible association with molar 
incisor hypomineralisation in Indian children. Eur Arch Paediatr Dent. 2015;16:441-7.

Oldin A, Lundgren J, Nilsson M, Norén JG, Robertson A. Traumatic dental injuries among children aged $0-17$ years in the BITA study - a longitudinal Swedish multicenter study. Dent Traumatol. 2015;31(1):9-17.

Sabokseir A, Golkari A, Sheiham A. Distinguishing between enamel fluorosis and other enamel defects in permanent teeth of children. PeerJ. 2016;4:e1745. https://doi.org/10.7717/peerj.1745.

Schmalfuss A, Stenhagen KR, Tveit AB, Crossner C-G, Espelid I. Canines are affected in 16-year-olds with molar-incisor hypomineralisation (MIH): an epidemiological study based on the Troms $\varnothing$ study: “Fit Futures". Eur Arch Paediatr Dent. 2016;17:107-13.

Schour I, Massler M. The development of human dentition. J Am Dent Assoc. 1941;28:1153-60.

Schwendicke F, Elhennawy K, Reda S, et al. Global burden of molar incisor hypomineralization. J Dent. 2018;68:10-8.

Silva MJ, Scurrah KJ, Craig JM, Manton DJ, Kilpatrick N. Etiology of molar incisor hypomineralization-a systematic review. Community Dent Oral Epidemiol. 2016;44:342-53.

Small BW, Murray JJ. Enamel opacities: prevalence, classification an aetiological considerations. J Dent. 1978;6:33-42.

Teixeira RJPB, Andrade NS, Queiroz LCC, et al. Exploring the association between genetic and environmental factors and molar incisor hypomineralization: evidence from a twin study. Int J Paediatr Dent. 2018;28(:198-206.
Vieira AR, Kup E. On the Etiology of Molar-Incisor Hypomineralization. Caries Res. 2016;50:166-9.

Weerheijm KL, Jälevik B, Alaluusua S. Molar-Incisor hypomineralisation. Caries Res. 2001;35:390-1.

Weerheijm KL, Duggal M, Mejàre I, et al. Judgement criteria for Molar Incisor Hypomineralisation (MIH) in epidemiologic studies: a summary of the European meeting on MIH held in Athens, 2003. Eur J Paed Dent. 2003;4:110-3.

Wong HM, McGrath C, Lo EC, King NM. Photographs as a means of assessing developmental defects of enamel. Community Dent Oral Epidemiol. 2005;33:438-46.

Wong HM, Wen YF, King NM, Patrick C, McGrath J. Longitudinal changes in developmental defects of enamel. Community Dent Oral Epidemiol. 2016;44:255-62.

Zhao D, Dong B, Yu D, Ren Q, Sun Y. The prevalence of molar incisor hypomineralization: evidence from 70 studies. Int J Paediatr Dent. 2018;28:170-9.

Publisher's Note Springer Nature remains neutral with regard to jurisdictional claims in published maps and institutional affiliations. 